\title{
Software security characteristics for function point analysis
}

\begin{abstract}
Software cost estimation (SCE) has been an important but difficult task since the beginning of the computer era. It considers list of parameters to estimate accurately the software cost. However, security cost is always excluded in most of the parametric cost estimation models. It is because of the security aspect is normally considered late in the software development. To overcome this problem, we proposed an enhancement to one of the parametric estimation models, which is function point analysis (FPA), to address the security concerns. The enhancement suggests potential software security characteristics during system development life cycle (SDLC). These characteristics are then integrated into FPA calculation to encounter the security cost estimation. This paper also points to the validation of a survey findings and calibration of the FPA calculation.
\end{abstract}

Keyword: Function point analysis; Security cost estimation; Software cost estimation; Software security characteristics 